\title{
Analytical Modeling of Current Overshoot in Oxide-Based Resistive Switching Memory (RRAM)
}

\author{
Stefano Ambrogio, Member, IEEE, Valerio Milo, ZhongQiang Wang, Simone Balatti, \\ and Daniele Ielmini, Senior Member, IEEE
}

\begin{abstract}
Current overshoot due to parasitic capacitance during set transition represents a major concern for controlling the resistance and current consumption in resistive switching memory (RRAM) arrays. In this letter, the impact of current overshoot on the low-resistance state (LRS) is evaluated by means of experiments on one-transistor/one-resistor structures of $\mathrm{HfO}_{2}$ RRAM. We develop a physics-based analytical model, able to calculate the LRS resistance and the corresponding reset current by a closed-form formula. The model allows predicting the current overshoot impact for any value of compliance current, set voltage, and parasitic capacitance.
\end{abstract}

Index Terms-Resistive switching memory, RRAM, capacitive overshoot, reliability, analytical modeling, non volatile memory.

\section{INTRODUCTION}

$\mathbf{R}$ ESISTIVE switching memory (RRAM) is a promising candidate for next-generation non-volatile memory and storage class memory [1]. RRAM offers high speed [2], high endurance [3] and good scaling thanks to atomic-scale conductive filament (CF) [4]. Reliability of RRAM is mostly affected by switching variability [5], low-frequency noise resulting in distribution broadening [6] and current overshoot during set transition [7]-[15]. The latter arises due to the parasitic capacitance $\mathrm{C}_{P}$, causing an additional discharge/charge current in the RRAM device even when the compliance current $\mathrm{I}_{C}$ is limited by a series transistor. Since $\mathrm{C}_{P}$ contains contributions from the bitline/wordline capacitances, overshoot effects play a major role in the power consumption and reliability of large RRAM arrays. Therefore, predicting and controlling the impact of the current overshoot is essential in the design of RRAM arrays.

In this letter, we present an analytical formula for the impact of $\mathrm{C}_{P}$ on resistance and reset current $\mathrm{I}_{\text {reset }}$. The model predicts

Manuscript received June 25, 2016; revised August 1, 2016; accepted August 9, 2016. Date of publication August 24, 2016; date of current version September 23, 2016. This work was supported by the European Research Council under Consolidator Grant ERC-2014-CoG-648635-RESCUE. The review of this letter was arranged by Editor L. Selmi.

S. Ambrogio, V. Milo, and D. Ielmini are with the Dipartimento di Elettronica, Informazione e Bioingegneria, Italian Universities Nanoelectronics Team, Politecnico di Milano, 20133 Milan, Italy (e-mail: daniele.ielmini@polimi.it).

Z. Wang was with the Dipartimento di Elettronica, Informazione e Bioingegneria and Italian Universities Nanoelectronics Team, Politecnico di Milano, 20133 Milano, Italy. He is now with North eastern Normal University, Changchun, People Republic of China.

S. Balatti was with the Dipartimento di Elettronica, Informazione e Bioingegneria and Italian Universities Nanoelectronics Team, Politecnico di Milano, 20133 Milan, Italy. He is now with Intermolecular, Inc., San Jose, CA 95134 USA.

Color versions of one or more of the figures in this letter are available online at http://ieeexplore.iee.org.

Digital Object Identifier 10.1109/LED.2016.2600574
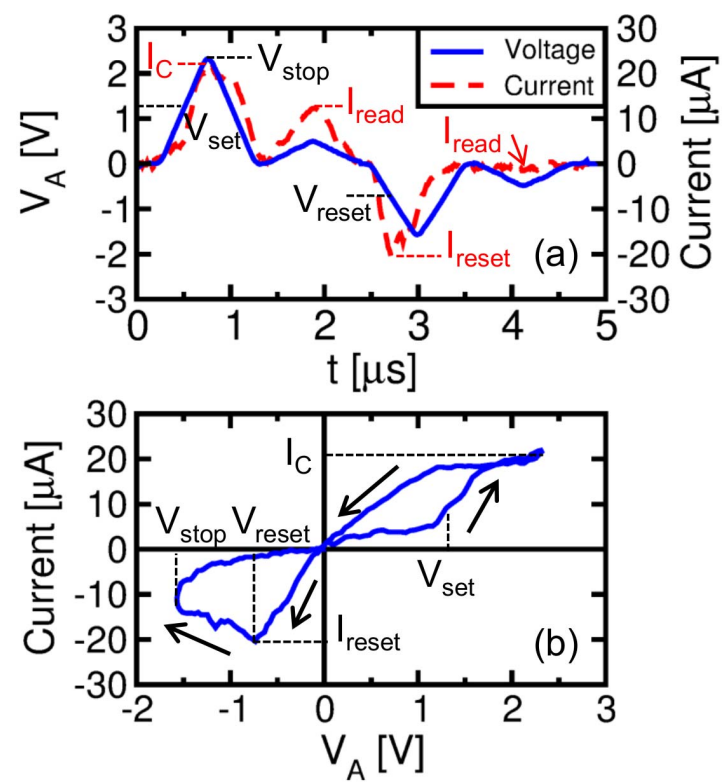

Fig. 1. Voltage and current experimental measurements for one cycle including set and reset processes and the corresponding read pulses (a), and the corresponding I-V curve (b). Definitions of $\mathrm{V}_{\text {set }}, \mathrm{V}_{\text {reset }}, \mathrm{V}_{\text {stop }}, \mathrm{I}_{C}$ and $\mathrm{I}_{\text {reset }}$ are reported in the figure.

the impact of the set voltage and $\mathrm{I}_{C}$ on overshoot. Our results support the ability of the model to accurately predict overshoot effects for RRAM at any arbitrary value of $C_{P}$, thus providing a valuable tool to evaluate the impact on large RRAM arrays.

\section{EXPERIMENTAL RESULTS}

We characterized switching and overshoot effects in Si-doped $\mathrm{HfO}_{x}$ RRAMs with a TiN bottom electrode (BE) and a Ti top electrode (TE) [16]. Devices were integrated in a 1-transistor/1-resistor (1T1R) structure to provide control of $\mathrm{I}_{C}$ and minimize $\mathrm{C}_{P}$ in parallel to the RRAM device. The parasitic capacitance includes contributions from the drain capacitance of the transistor, and the connecting metal line between transistor and RRAM. In a RRAM array, $\mathrm{C}_{P}$ might also include the bitline/wordline capacitance. Experiments were conducted by applying voltage pulses to the gate electrode and the TE with an arbitrary waveform generator, while TE voltage and current in the MOSFET were monitored by an oscilloscope. Fig. 1 shows the typical result of the pulsed characteristics of our RRAM device. Fig. 1a shows the applied TE voltage during an individual cycle including set and reset pulses, each followed by a read pulse to probe the resistance state. Each pulse had a duration $\mathrm{t}_{P}=1 \mu \mathrm{s}$. 

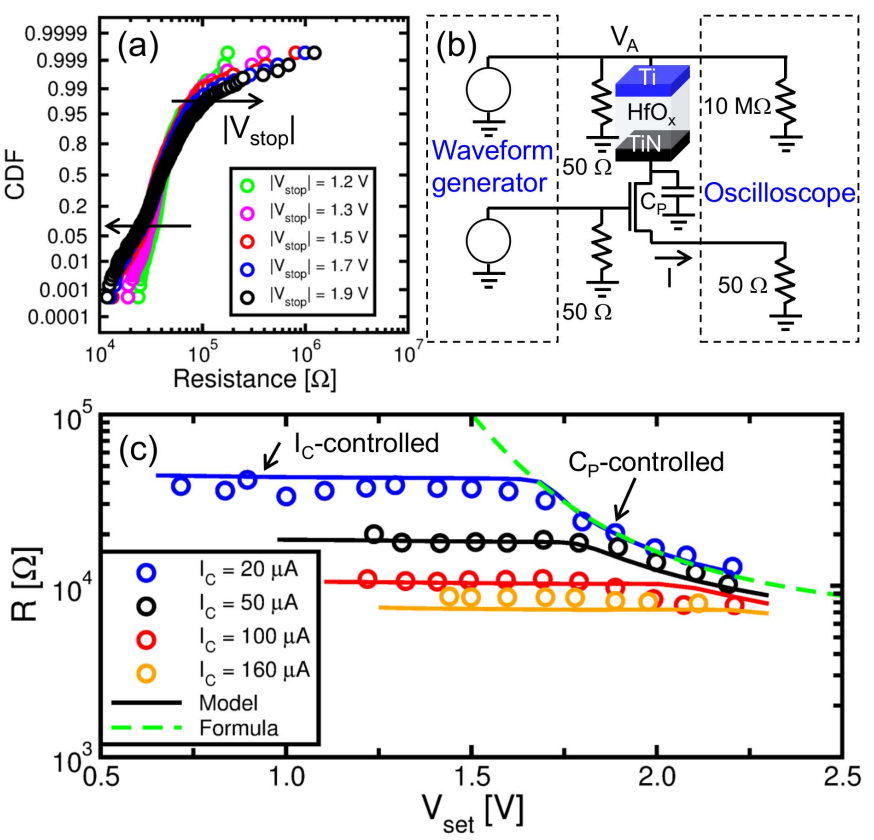

Fig. 2. Experimental cumulative distributions of LRS for increasing $\left|\mathrm{V}_{\text {stop }}\right|$ (a), schematic illustration of the 1T1R circuit with parasitic capacitance (b), and average LRS resistance as a function of $\mathrm{V}_{\text {set }}$ from data and calculations. For high $\left|\mathrm{V}_{\text {stop }}\right|$, a low resistance tail due to parasitic overshoot appears in (a), together with a high resistance tail due to non-switching events. Calculations from a device compact model and the analytical Eq. (5) well agree with data in the $\mathrm{C}_{P}$-controlled region in (c).

The dashed line displays the measured current revealing set/reset processes. A set transition to the low-resistance state (LRS) appears during the set pulse, followed by a read pulse of $0.5 \mathrm{~V}$. The subsequent reset pulse causes the reset transition to the high resistance state (HRS), measured by a second voltage pulse of $-0.5 \mathrm{~V}$. Note the significantly different $\mathrm{I}_{\text {read }}$ after set $\left(\mathrm{I}_{\text {read }} \approx 10 \mu \mathrm{A}\right)$ and after reset $\left(\mathrm{I}_{\text {read }} \approx 0\right)$. Fig. $1 \mathrm{~b}$ shows the corresponding I-V curve extracted from Fig. 1a. $\mathrm{I}_{C}$ was equal to $20 \mu \mathrm{A}$ in the experiment in Fig. 1b by control of the gate voltage $\mathrm{V}_{G}=1.2 \mathrm{~V}$ in the transistor. The set voltage $\mathrm{V}_{\text {set }} \approx 1.3 \mathrm{~V}$ is marked by the transition to the LRS. Reset transition takes place at about $-0.8 \mathrm{~V}$ with a reset current $\mathrm{I}_{\text {reset }} \approx 20 \mu \mathrm{A}$, close to $\mathrm{I}_{C}$ [17]. Note that $\mathrm{V}_{\text {set }}$ corresponds to the $\mathrm{V}_{A}$ able to induce a set transition in the device, thus it is applied to the whole 1T1R structure. We used triangular pulses to extract $\mathrm{V}_{\text {set }}$ and $\mathrm{V}_{\text {reset }}$, however, similar results could be obtained for pulses with other shapes.

\section{PARASITIC OVERShOOT}

We cycled RRAM cells for 1000 cycles, then we extracted the cumulative distribution function (CDF) of the LRS resistance. Results are shown in Fig. 2a, for increasing $\left|V_{\text {stop }}\right|$. The $\mathrm{CDF}$ shows tails at high and low resistance, both increasing with $\left|V_{\text {stop }}\right|$. The high resistance tail is due to non-switching events, where the device could not switch, or could switch only partially, due to the relatively large value of $\mathrm{V}_{\text {set }}$. As $\left|V_{\text {stop }}\right|$ increases, the reset transition results in a deeper HRS with a relatively large $\mathrm{V}_{\text {set }}$, which thus causes increasing high-resistance tail in the LRS distribution. On the other hand, the low resistance tail is due to the current overshoot

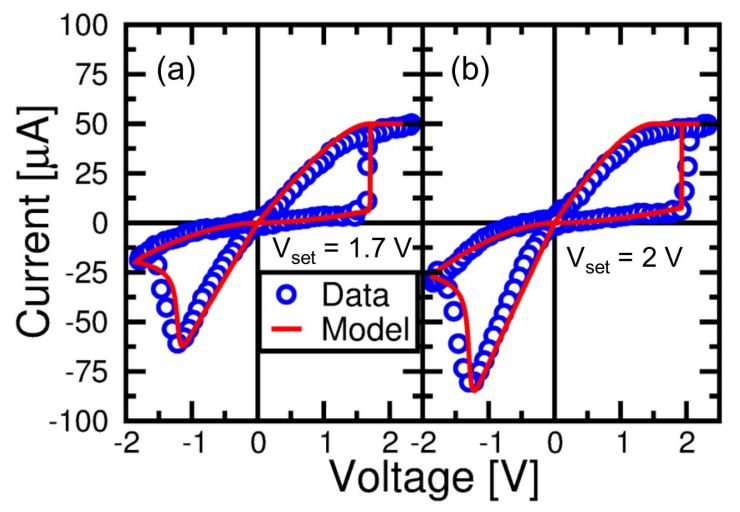

Fig. 3. Experimental and calculated $\mathrm{I}-\mathrm{V}$ curves for different $\mathrm{V}_{\text {set }}=1.7 \mathrm{~V}$ (a) and $2 \mathrm{~V}$ (b). Increasing $\mathrm{V}_{\text {set }}$ leads to decreasing LRS resistance hence higher $\left|\mathrm{I}_{\text {reset }}\right|$.

resulting from $\mathrm{C}_{P}$ connected to the node between RRAM and MOSFET, as shown in Fig. 2b. The capacitive current across $C_{P}$ causes a transient excess current higher than the nominal $\mathrm{I}_{C}$ during set transition. The capacitive current is due to the sudden increase of the potential across $C_{P}$ as the $1 T 1 \mathrm{R}$ voltage divider is changed by the set transition. The extra-current induced by $\mathrm{C}_{P}$ charging, in combination with the relatively large $\mathrm{V}_{\text {set }}$ accelerating ion migration, leads to a CF overgrowth, with a subsequent lower LRS resistance in correspondence of the LRS low-resistance tail. As $\left|V_{\text {stop }}\right|$ increases, HRS resistance and $\mathrm{V}_{\text {set }}$ increase, thus causing larger overshoot effects due to the exponential increase of ion migration rate with voltage [17], [18]. The low-resistance tail at increasing $\mathrm{V}_{\text {stop }}$ in Fig. 2a can thus be attributed to the overshoot arising for large $\mathrm{V}_{\text {set }}$.

Fig. 2c shows the correlation between the average value of the LRS resistance as a function of $V_{\text {set }}$ in the preceding set operation, for $\mathrm{I}_{C}=20 \mu \mathrm{A}, 50 \mu \mathrm{A}, 100 \mu \mathrm{A}$ and $160 \mu \mathrm{A}$. At low $\mathrm{V}_{\text {set }}<1.5 \mathrm{~V}$, the LRS resistance shows a constant value, which increases at decreasing $\mathrm{I}_{C}$, namely an $\mathrm{I}_{C}$-controlled regime. For higher $\mathrm{V}_{\text {set }}$, the LRS resistance decreases with $\mathrm{V}_{\text {set }}$ revealing the effect of the current overshoot, namely the $\mathrm{C}_{P}$-controlled regime.

Results are reproduced by simulations of the set transition in a 1T1R structure using a compact model of the RRAM [19] assuming $\mathrm{C}_{P}=30 \mathrm{fF}$. The compact model in [19] was demonstrated for RRAM devices under various set/reset conditions and for simple RRAM circuits [20], [21]. In the simulations, $\mathrm{V}_{\text {set }}$ is adjusted by assuming variable resistance in the HRS, which is naturally obtained in the experiment thanks to the stochastic switching in our RRAM [5]. Fig. 3 reports the impact of capacitive overshoot at variable $\mathrm{V}_{\text {set }}$, showing the measured and calculated I-V curves for relatively low $\mathrm{V}_{\text {set }}=1.7 \mathrm{~V}$ (a) and high $\mathrm{V}_{\text {set }}=2 \mathrm{~V}$ (b). In both cases, $\mathrm{I}_{C}$ was fixed to $\mathrm{I}_{C}=50 \mu \mathrm{A}$. For $\mathrm{V}_{\text {set }}=1.7 \mathrm{~V}$, current overshoot is negligible as the $\mathrm{CF}$ growth time is comparable or longer than the $\mathrm{RC}$ constant, thus the voltage across $\mathrm{C}_{P}$ is readjusted before or during $\mathrm{CF}$ growth. On the other hand, Fig. $3 \mathrm{~b}$ shows that for $\mathrm{V}_{\text {set }}=2 \mathrm{~V}$, LRS resistance reaches a lower value due to the $\mathrm{CF}$ growth time being shorter than the $\mathrm{RC}$ time. The lower resistance is reflected by a larger $\mathrm{I}_{\text {reset }}$ in the subsequent reset operation, further supporting the evidence 
of a CF overgrowth. Since $\mathrm{I}_{C} \approx \mathrm{I}_{\text {reset }}$, an effective overshoot current during set transition around $80 \mu \mathrm{A}$ can be estimated from Fig. 3b. The compact model accounts for the observed values of LRS resistance and $\mathrm{I}_{\text {reset }}$.

\section{Analytical Model of Overshoot EfFects}

To predict the LRS resistance as a function of $\mathrm{V}_{\text {set }}$ and $\mathrm{C}_{P}$, we consider the growth rate of the $\mathrm{CF}$ diameter $\phi$ given by [17]:

$$
\frac{d \phi}{d t}=A e^{-\frac{E_{A}-\alpha q V}{k\left(T_{0}+\frac{V 2}{8 \rho k_{t h}}\right)}},
$$

where $\mathrm{A}=200 \mathrm{~ms}^{-1}$ is a pre-exponential factor, $\mathrm{E}_{A}=1.28 \mathrm{eV}$ is the energy barrier for ion migration, $\alpha=0.3$ [22] is a barrier lowering coefficient, $\mathrm{k}$ is the Boltzmann constant and $\mathrm{V}$ is the applied voltage. Joule heating is described by the denominator in the exponent, where $\mathrm{T}_{0}$ is the ambient temperature, $\rho$ and $\mathrm{k}_{t h}$ are the effective resistivity and thermal conductivity, with values $\rho=1.97 \mathrm{~m} \Omega \mathrm{cm}$ and $k_{t h}=7 \mathrm{Wm}^{-1} K^{-1}$. The exponential dependence of switching speed on voltage in Eq. (1) is similar to other proposed models in the literature for migration-based switching in RRAM [17], [22]-[28]. Different models describing CF restoration during set transition lead to the same qualitative behavior, provided that an exponential voltage acceleration is assumed for the set process. Assuming that the voltage across the device is kept constant by $\mathrm{C}_{P}$ during $\mathrm{CF}$ growth, we can estimate the $\mathrm{CF}$ diameter $\phi_{L R S}$ after set process by integrating Eq. (1), namely:

$$
\phi_{L R S}=\int_{0}^{\tau_{s e t}} \frac{d \phi}{d t} d t=A e^{-\frac{E_{A}-\alpha q V_{s e t}}{k\left(T_{0}+\frac{V_{s e t}^{2}}{\left.8 \rho k_{t h}\right)}\right.}} \tau_{\text {set }},
$$

where $\tau_{\text {set }}$ is the effective set time. The latter can be approximated by the $\mathrm{RC}$ time constant in the $\mathrm{C}_{P}$-controlled overshoot regime in Fig. 2c, namely:

$$
\tau_{\text {set }}=R C_{P},
$$

where $\mathrm{R}$ is the LRS resistance which can be calculated approximating the $\mathrm{CF}$ shape by a cylinder, thus leading to:

$$
R=\rho \frac{L}{\frac{\pi}{4} \phi_{L R S}^{2}},
$$

where $\mathrm{L}=5 \mathrm{~nm}$ is the $\mathrm{CF}$ length. By substituting Eq. (3) and (4) in Eq. (2), we obtain

$$
R=\left(\frac{4 \rho L}{\pi A^{2}}\right)^{1 / 3} e^{\frac{E_{A}-\alpha q V}{k\left(T_{0}+\frac{V_{s e t}^{2}}{\left.8 \rho k_{t h}\right)}\right.}} C_{P}^{-2 / 3},
$$

which directly yields $\mathrm{R}$ as a function of $\mathrm{C}_{P}$ and $\mathrm{V}_{\text {set }}$ in the $\mathrm{C}_{P}$ controlled regime. From Eq. (5), $\mathrm{I}_{\text {reset }}$ can be estimated as $\mathrm{I}_{\text {reset }}=\mathrm{V}_{\text {reset }} / \mathrm{R}$, where $\mathrm{V}_{\text {reset }}$ is a characteristic constant for a given pulse width [17]. It can be noticed that $\mathrm{R}$ does not depend on $\mathrm{I}_{C}$, but follows a universal behavior, in agreement with data in Fig. 2c. The results of the analytical formula are plotted in Fig. 2c, showing a good agreement with data and with the compact model in the $\mathrm{C}_{P}$-controlled region. Eq. (5) applies to the $C_{P}$-controlled region, where the overshoot transient takes place in a time $\tau_{\text {set }}$ which is generally
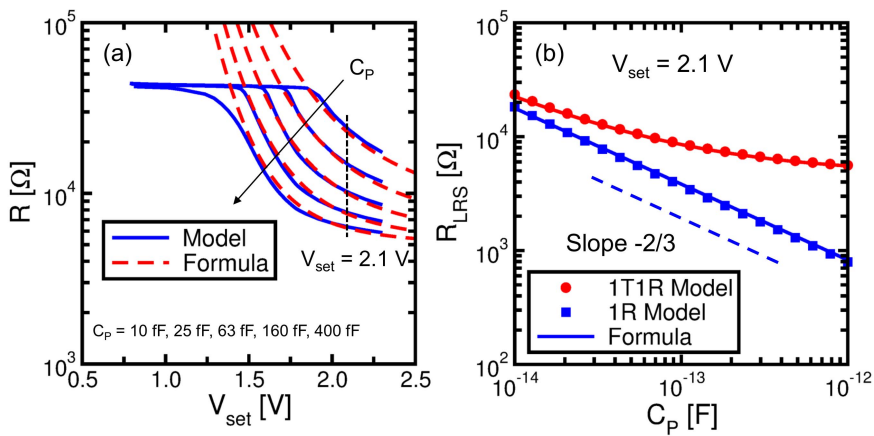

Fig. 4. Calculated R from the compact model [19] and from the formula in Eq. (5) as a function of $\mathrm{V}_{\text {set }}$ for different $\mathrm{C}_{P}$, from $10 \mathrm{fF}$ to $400 \mathrm{fF}$ (a). (b) shows the $1 \mathrm{~T} 1 \mathrm{R}$ and $1 \mathrm{R}$ resistances versus $\mathrm{C}_{P}$ at a constant $\mathrm{V}_{\text {set }}=2.1 \mathrm{~V}$, as shown in (a). Results show a complete agreement, with a slope $-2 / 3$ of the $1 \mathrm{R}$ resistance.

much smaller than the actual pulse width $\mathrm{t}_{P}$. For instance, for $\mathrm{R}=10 \mathrm{k} \Omega$ and $\mathrm{C}_{P}=100 \mathrm{fF}$, one obtains $\tau_{\text {set }}=1 \mathrm{~ns}$, hence much shorter than $\mathrm{t}_{P}$. Therefore, we do not expect any significant change of Eq. (5) at variable $\mathrm{t}_{P}$, at least for $\mathrm{t}_{P}>1 \mathrm{~ns}$.

\section{DISCUSSION}

In our work the parasitic capacitance $\mathrm{C}_{P}=30 \mathrm{fF}$ is consistent with the drain-capacitance of the transistor in the $1 \mathrm{~T} 1 \mathrm{R}$ with possible contributions from the metal interconnect. On the other hand, the capacitive load in high-density memory circuits might be significantly larger than $\mathrm{C}_{P}=30 \mathrm{fF}$ as a result of, e.g., long bitlines in a crossbar array. To assess the impact of $\mathrm{C}_{P}$, Fig. 4a shows the calculated $\mathrm{R}$ as a function of $\mathrm{V}_{\text {set }}$ for increasing $\mathrm{C}_{P}$, from $10 \mathrm{fF}$ to $400 \mathrm{fF}$. Results from Eq. (5) are compared to simulations as in Fig. 2 and 3. The onset of the $\mathrm{C}_{P}$-controlled regime is anticipated at increasingly low $\mathrm{V}_{\text {set }}$ for increasing $\mathrm{C}_{P}$, as a result of the $\mathrm{C}_{P}{ }^{-2 / 3}$ dependence in Eq. (5). Note the saturation of the LRS resistance equal to $4.7 \mathrm{k} \Omega$ for high $\mathrm{V}_{\text {set }}$ due to the series resistance of the transistor. The formula accounts very well for the $\mathrm{R}$ behavior in the $\mathrm{C}_{P}$ regime, even for a wide $\mathrm{C}_{P}$ range of almost 2 decades.

To further assess the validity of Eq. (5), we evaluated the LRS resistance as a function of $C_{P}$ for a constant $\mathrm{V}_{\text {set }}=2.1 \mathrm{~V}$, as indicated in Fig. 4a. Fig. $4 \mathrm{~b}$ shows the $1 T 1 \mathrm{R}$ compact model resistance and the corresponding $1 \mathrm{R}$ compact model resistance of the RRAM (i.e., without the series resistance of the transistor) as a function of $\mathrm{C}_{P}$. The computed results from Eq. (5) are also shown for comparison, in good agreement with simulations. In particular, the $1 \mathrm{R}$ resistance shows a slope $-2 / 3$ on the $\log -\log$ plot, as expected from the $\mathrm{C}_{P}{ }^{-2 / 3}$ term in Eq. (5).

\section{CONCLUSION}

We studied current overshoot affecting the set transition at high $\mathrm{V}_{\text {set }}$ in RRAM devices. An analytical formula is presented to easily predict LRS resistance as a function of $\mathrm{V}_{\text {set }}, \mathrm{C}_{P}$ and other microscopic parameters in the device. Our analysis suggests that current overshoot can be minimized by accurately controlling $\mathrm{V}_{\text {set }}$ and its variability, and by limiting $\mathrm{C}_{P}$ and the switching speed of the RRAM. 


\section{REFERENCES}

[1] H.-S. P. Wong, H.-Y. Lee, S. Yu, Y.-S. Chen, Y. Wu, P.-S. Chen, B. Lee, F. T. Chen, and M.-J. Tsai, "Metal-oxide RRAM," Proc. IEEE, vol. 100, no. 6, pp. 1951-1970, Jun. 2012, doi: 10.1109/JPROC.2012. 2190369.

[2] Y. S. Chen, H. Y. Lee, P. S. Chen, P. Y. Gu, C. W. Chen, W. P. Lin, W. H. Liu, Y. Y. Hsu, S. S. Sheu, P. C. Chiang, W. S. Chen, F. T. Chen, C. H. Lien, and M.-J. Tsai, "Highly scalable hafnium oxide memory with improvements of resistive distribution and read disturb immunity," in IEDM Tech. Dig., Dec. 2009, pp. 1-4.

[3] M.-J. Lee et al., "A fast, high-endurance and scalable non-volatile memory device made from asymmetric $\mathrm{Ta}_{2} \mathrm{O}_{5-x} / \mathrm{TaO}_{2-x}$ bilayer structures," Nature Mater, vol. 10, pp. 625-630, Aug. 2011.

[4] J. Shin et al., "Effect of program/erase speed on switching uniformity in filament-type RRAM," IEEE Electron Device Lett., vol. 32, no. 7, pp. 958-960, Jul. 2011.

[5] S. Ambrogio, S. Balatti, A. Cubeta, A. Calderoni, N. Ramaswamy, and D. Ielmini, "Statistical fluctuations in $\mathrm{HfO}_{x}$ resistive-switching memory: Part I-Set/reset variability," IEEE Trans. Electron Devices, vol. 61, no. 8, pp. 2912-2919, Aug. 2014.

[6] S. Ambrogio, S. Balatti, V. McCaffrey, D. C. Wang, and D. Ielmini, "Noise-induced resistance broadening in resistive switching memoryPart II: Array statistics," IEEE Trans. Electron Devices, vol. 62, no. 11, pp. 3812-3819, Nov. 2015.

[7] K. Kinoshita, K. Tsunoda, Y. Sato, H. Noshiro, S. Yagaki, M. Aoki, and Y. Sugiyama, "Reduction in the reset current in a resistive random access memory consisting of $\mathrm{NiO}_{x}$ brought about by reducing a parasitic capacitance," Appl. Phys. Lett., vol. 93, no. 3, p. 033506, 2008.

[8] T. Sakamoto, M. Tada, M. Miyamura, N. Banno, K. Okamoto, N. Iguchi, and H. Hada, "Impact of overshoot current on set operation of atom switch," Jpn. J. Appl. Phys., vol. 53, no. 4S, p. 04ED07, 2014.

[9] D. C. Sekar, B. Bateman, U. Raghuram, S. Bowyer, Y. Bai, M. Calarrudo, P. Swab, J. Wu, S. Nguyen, N. Mishra, R. Meyer, M. Kellam, B. Haukness, C. Chevallier, H. Wu, H. Qian, F. Kreupl, and G. Bronner, "Technology and circuit optimization of resistive RAM for low-power, reproducible operation," in IEDM Tech. Dig., Dec. 2014, pp. 28.3.1-28.3.4.

[10] D. S. Byeon, C.-W. Yoon, H.-K. Park, Y.-K. Lee, H.-J. Kwon, Y.-T. Lee, K.-S. Kim, Y.-Y. Joo, I.-G. Baek, Y.-B. Kim, J.-D. Choi, K.-H. Kyung, and J.-H. Choi, "Disturbance-suppressed ReRAM write algorithm for high-capacity and high-performance memory," in Proc. NVMTS, Oct. 2014, pp. 1-4.

[11] J. Song, D. Lee, J. Woo, Y. Koo, E. Cha, S. Lee, J. Park, K. Moon, S. H. Misha, A. Prakash, and H. Hwang, "Effects of RESET current overshoot and resistance state on reliability of RRAM," IEEE Electron Device Lett., vol. 35, no. 6, pp. 636-638, Jun. 2014.

[12] S. Yu, X. Guan, and H.-S. P. Wong, "Understanding metal oxide RRAM current overshoot and reliability using kinetic Monte Carlo simulation," in IEDM Tech. Dig., Dec. 2012, pp. 26.1.1-26.1.4.

[13] D. C. Gilmer, G. Bersuker, H.-Y. Park, C. Park, B. Butcher, W. Wang, P. D. Kirsch, and R. Jammy, "Effects of RRAM stack configuration on forming voltage and current overshoot," in Proc. IMW, May 2011, pp. 1-4.

[14] P. Shrestha, D. Nminibapiel, J. P. Campbell, K. P. Cheung, H. Baumgart, S. Deora, and G. Bersuker, "Dependence of the filament resistance on the duration of current overshoot," in Proc. IIRW, Oct. 2013, pp. 55-58.
[15] Y. Fang, Y. Cai, Z. Wang, Z. Yu, X. Yang, and R. Huang, "Influence of selector-introduced compliance current on $\mathrm{HfO}_{x}$ RRAM switching operation," in Proc. NVMTS, Oct. 2015, pp. 1-3.

[16] S. Balatti, S. Ambrogio, Z. Wang, S. Sills, A. Calderoni, N. Ramaswamy, and D. Ielmini, "Voltage-controlled cycling endurance of $\mathrm{HfO}_{x}$-based resistive-switching memory," IEEE Trans. Electron Devices, vol. 62, no. 10 , pp. $3365-3372$, Oct. 2015.

[17] D. Ielmini, "Modeling the universal set/reset characteristics of bipolar RRAM by field- and temperature-driven filament growth," IEEE Trans. Electron Devices, vol. 58, no. 12, pp. 4309-4317, Dec. 2011.

[18] S. Larentis, F. Nardi, S. Balatti, D. C. Gilmer, and D. Ielmini, "Resistive switching by voltage-driven ion migration in bipolar RRAM-Part II: Modeling," IEEE Trans. Electron Devices, vol. 59, no. 9, pp. 2468-2475, Sep. 2012.

[19] S. Ambrogio, S. Balatti, D. C. Gilmer, and D. Ielmini, "Analytical modeling of oxide-based bipolar resistive memories and complementary resistive switches," IEEE Trans. Electron Devices, vol. 61, no. 7, pp. 2378-2386, Jul. 2014.

[20] S. Ambrogio, S. Balatti, V. Milo, R. Carboni, Z.-Q. Wang, A. Calderoni, N. Ramaswamy, and D. Ielmini, "Neuromorphic learning and recognition with one-transistor-one-resistor synapses and bistable metal oxide RRAM," IEEE Trans. Electron Devices, vol. 63, no. 4, pp. 1508-1515, Apr. 2016.

[21] S. Balatti, S. Ambrogio, and D. Ielmini, "Normally-off logic based on resistive switches-Part I: Logic gates," IEEE Trans. Electron Devices, vol. 62, no. 6, pp. 1831-1838, Jun. 2015.

[22] P.-Y. Chen and S. Yu, "Compact modeling of RRAM devices and its applications in 1T1R and 1S1R array design," IEEE Trans. Electron Devices, vol. 62, no. 12, pp. 4022-4028, Dec. 2015.

[23] P. Huang et al., "A physics-based compact model of metal-oxide-based RRAM DC and AC operations," IEEE Trans. Electron Devices, vol. 60, no. 12, pp. 4090-4097, Dec. 2013.

[24] L. Larcher, F. M. Puglisi, P. Pavan, A. Padovani, L. Vandelli, and G. Bersuker, "A compact model of program window in $\mathrm{HfO}_{\mathrm{x}}$ RRAM devices for conductive filament characteristics analysis," IEEE Trans. Electron Devices, vol. 61, no. 8, pp. 2668-2673, Aug. 2014.

[25] G. Bersuker, D. C. Gilmer, D. Veksler, J. Yum, H. Park, S. Lian, L. Vandelli, A. Padovani, L. Larcher, K. McKenna, A. Shluger, V. Iglesias, M. Porti, M. Nafria, W. Taylor, P. D. Kirsch, and R. Jammy, "Metal oxide RRAM switching mechanism based on conductive filament microscopic properties," in IEDM Tech. Dig., Dec. 2010, pp. 19.6.1-19.6.4.

[26] R. Degraeve, A. Fantini, S. Clima, B. Govoreanu, L. Goux, Y.-Y. Chen, D. Wouters, P. Roussel, G. Kar, G. Pourtois, S. Cosemans, J. Kittl, G. Groeseneken, M. Jurczak, and L. Altimime, "Dynamic 'hour glass' model for SET and RESET in $\mathrm{HfO}_{2}$ RRAM," in VLSI Tech. Dig., Jun. 2012, pp. 75-76.

[27] F. M. Puglisi, P. Pavan, A. Padovani, and L. Larcher, "A compact model of hafnium-oxide-based resistive random access memory," in Proc. Int. Conf. IC Design Technol. (ICICDT), May 2013, pp. 85-88.

[28] H. Li, Z. Jiang, P. Huang, Y. Wu, H. Y. Chen, B. Gao, X. Y. Liu, J. F. Kang, and H.-S. P. Wong, "Variation-aware, reliabilityemphasized design and optimization of RRAM using SPICE model," in Proc. Design, Autom. Test Eur. Conf. Exhibit. (DATE), 2015, pp. $1425-1430$. 